\section{Co-Infecção HIV/HCV em pacientes de Botucatu e região}

\author{
HIV/HCV Co-Infection in Patients \\ from Botucatu and Region (Brazilian \\ cities)
}

Sílvia M. Corvino'

Rita M.S. Henriques ${ }^{2}$

Rejane M.T. Grotto ${ }^{2}$

Maria Inês de M.C. Pardini²

${ }^{1}$ Instituto Adolfo Lutz, Laboratório I de Sorocaba, SP

2 UNESP- Faculdade de Medicina, Hemocentro, Laboratório de Biologia

Molecular / Hepatites Virais, Botucatu, SP

\section{Resumo}

Devido à similaridade nas rotas de transmissão, a co-infecção HIV/HCV é freqüente, afetando em média 30 a $50 \%$ dos portadores de HIV. O presente estudo visou avaliar uma possível associação entre os subtipos do HIV e genótipos do HCV em pacientes co-infectados, com base na análise das freqüências em pacientes mono e co-infectados. Para determinação da freqüência dos subtipos HIV e genótipos HCV, foram analisados respectivamente 124 e 496 pacientes mono-infectados. O estudo da co-infecção foi realizado num grupo de 150 pacientes HIV positivos e esteve presente em $22(14,7 \%)$ dos pacientes. A freqüência dos subtipos do HIV-1 em mono-infectados foi: subtipo B (85,5\%), subtipo F (12,9\%) e recombinante $\mathrm{B} / \mathrm{F}(1,6 \%)$, enquanto nos genótipos HCV foi: $1 \mathrm{a}(25 \%), 1 \mathrm{~b}(29,4 \%), 1 \mathrm{a} /$ lb (3,6\%), 3a (35\%), 2 (1,8\%) e 5 (0,4\%). Nos co-infectados o padrão de distribuição dos subtipos HIV-1 é semelhante aos monoinfectados, ou seja, subtipo B $(85,0 \%)$, seguido do subtipo F (15,0\%). A distribuição de freqüência de genótipos HCV nos coinfectados foi: $1 \mathrm{a}(36,3 \%), 1 \mathrm{~b}(27,3 \%), 1 \mathrm{a} / 1 \mathrm{~b}$ $(9,1 \%)$ e $3 a(27,3 \%)$ mostrando um aumento de $10 \%$ na freqüência do genótipo 1 , queda de 7,7\% no genótipo 3 e ausência de outros genótipos. A análise estatística de associação entre os subtipos HIV e genótipos HCV (Goodman) mostrou que no genótipo 1 (HCV) ocorreu predominância do subtipo $\mathrm{B}$, enquanto no genótipo $3(\mathrm{HCV})$ a distribuição dos subtipos B e F (HIV-1) foi casual. Isto aponta para a necessidade de mais estudos desse grupo e um maior valor amostral.

Palavras-chave: Co-infecção HIV/HCV. Genótipos. Subtipos.

Correspondência: Sílvia M. Corvino. Rua Gino Cariola, 88. Jardim Paraíso, Botucatu, SP

CEP 18609-060. E-mail: silma_cor@yahoo.com.br 
Abstract

$\mathrm{HIV} / \mathrm{HCV}$ co-infection is a frequent event due to the similarity of the means of transmission of both viruses; $30-50 \%$ of HIV infected individuals are co-infected ${ }^{1}$. This paper assesses the possible association among HCV and HIV genotypes in co-infected patients based on frequency distribution in mono and co-infected patients. To determine HIV and HCV genotype frequency 124 and 496 respectively, mono infected patients were analyzed. The study of co-infection was performed in $150 \mathrm{HIV}$ positive patients and 22 (14.7\%) patients were found. The frequency of HIV-1 subtypes was 106 B subtype (85.5\%), $16 \mathrm{~F}$ (12.9\%), and $2 \mathrm{~B} / \mathrm{F}$ recombinant (1.6\%); HCV genotypes were 1a (25\%), $1 \mathrm{~b}(29.4 \%), 1 \mathrm{a} / 1 \mathrm{~b}(3.6 \%), 3 \mathrm{a}(35 \%)$, $2(1.8 \%)$ and $5(0.4 \%)$. The HCV genotype could not be determined in $6.3 \%$ of samples using the technique. The HIV-1 subtype distribution standard was B subtype $(85.0 \%)$ and F subtype $(15.0 \%)$ in mono-infected as in co-infected. The frequency distribution of HCV genotypes in co-infected was la (36.4\%), lb (27.3\%), $1 \mathrm{a} / \mathrm{lb}(9.1 \%)$ and $3 \mathrm{a}(27.3 \%)$. These results showed a $10 \%$ increase in frequency of 1a genotype, $7.7 \%$ decrease in 3 genotype and lack of other genotypes. The statistical analysis of association of HCV genotypes and HIV-1 subtypes (Goodman Test) showed a predominance of the B HIV subtype among HCV genotype 1, and among HCV genotype 3 the distribution of $B$ and F HIV subtypes was casual. These results suggest the need for further studies in this group and larger samples.

Keywords: Co-infection HIV/HCV. Genotype. Subtype.

\section{Introdução}

Devido à similaridade nas rotas de transmissão, a co-infecção HIV/HCV é relativamente freqüente, afetando 30 a $50 \%$ dos portadores de $\mathrm{HIV}^{1}$.

O HIV, um retrovírus, e o HCV, um flavivírus, são ambos vírus de RNA, têm distribuição mundial, conduzem à infecção subclínica crônica ${ }^{2}$, apresentam genomas altamente polimórficos como conseqüência de altos níveis de mutações ${ }^{3}$, e podem ser diagnosticados utilizando-se métodos sorológicos e moleculares ${ }^{4-6}$.

A co-infecção induz a um pior prognóstico de ambas as infecções, dificultando a resposta imunológica do hospedeiro. Neste contexto, a debilitação imunológica causada pelo HIV acaba conduzindo à evolução da infecção pelo HCV e rápido curso natural da infecção, reduzindo de aproximadamente 30 para sete anos o comprometimento hepático com aparecimento de cirrose e hepatocarcinoma, que constituem causa de morte em pacientes infectados com HIV $\mathrm{H}^{7,8}$.

Do mesmo modo, o HCV parece diminuir o tempo para o aparecimento de aids e morte, dificultando a reconstituição do sistema imunológico e elevando o risco de hepatotoxicidade. O comprometimento hepático decorrente da infecção pelo HCV é agravado pela utilização de drogas integrantes da Terapia Anti-retroviral de Alta Atividade (HAART) ${ }^{9}$, que são hepatotóxicas. O risco de hepatotoxicidade induzida por drogas em co-infectados é de três a quatro vezes superior aos monoinfectados, o que parece estar diretamente relacionado à severidade da doença hepática $^{10}$.

Tanto o HCV como o HIV podem ser subdividos em diferentes genótipos e subtipos, que apresentam diferentes níveis de divergência das seqüências originais do vírus, dependendo da região genômica estudada, resultantes de erros durante a replicação viral causadas pela ausência de atividade de reparo da enzima RNA polimerase $^{11-13}$ 
O efeito dos diferentes genótipos do HCV na co-infecção é controverso. Alguns autores afirmam que a infecção por determinados ou múltiplos genótipos do $\mathrm{HCV}$ constitui um obstáculo ao tratamento pela HAART $^{14,15}$, enquanto outros referem não existir nenhuma influência dos genótipos do HCV na progressão da doença ${ }^{16,17}$.

O presente estudo visou avaliar uma possível associação entre os subtipos do HIV e genótipos do HCV em pacientes co-infectados, com base na análise das freqüências em pacientes mono e co-infectados.

\section{Material e Métodos}

\section{Critérios de inclusão}

Para determinação da freqüência dos genótipos HIV e HCV foram analisados respectivamente 124 e 496 pacientes monoinfectados, de ambos os sexos, maiores de 18 anos, de maneira aleatória, acompanhados rotineiramente nos ambulatórios: 1) Doenças Tropicais e Diagnóstico por Imagem e 2) Hepato-Hemocentro, da Faculdade de Medicina de Botucatu, Unesp.

O estudo da co-infecção foi realizado num grupo de 150 pacientes HIV positivos de Botucatu e região, escolhidos também de maneira aleatória, dos mesmos ambulatórios.

Este estudo teve aprovação do Comitê de Ética em Pesquisa da Faculdade de Medicina de Botucatu, UNESP.

\section{Metodologia}

O RNA viral do HCV foi detectado pela metodologia $R T$-PCR, utilizando-se Kit diagnóstico Amplicor da Roche ${ }^{\circledR}$, seguindo as especificações do fabricante.

A genotipagem do HCV foi realizada analisando-se a região ultraconservada 5' UTR com o Kit LiPA (Line Probe Assay Versant HCV Genotype - Bayer ${ }^{\mathrm{TM}}$ ), segundo instruções do fabricante.

A subtipagem para o HIV-1 foi realizada pela metodologia de seqüenciamento automático de DNA in house abaixo descrita.
Extração de DNA: Kit QIAmp DNA Blood (Quiagen) segundo as especificações do fabricante.

A amplificação foi realizada por Nested PCR da região C2 da gp120 do envelope viral. A primeira reação em Cadeia da Polimerase (PCR) foi realizada utilizando uma mistura contendo 1X Buffer, 2,5 mM de $\mathrm{MgCl}_{2}$, 0,4mM dNTP, 0,2pmol dos primers ED5 (5' - ATGGGATCAAAGCCTAAAGCCATGTG 3'), e ED12 (5' - AGTGCTTCCTGCTGCT CCCAAGAACCCAAG - 3') ${ }^{18}, 2,5 \mathrm{U}$ de Taq Recombinant DNA polimerase (Invitrogen) e 150-200ng de DNA, num volume total de reação de $50 \mu \mathrm{L}$. A reação procedeu-se com uma desnaturação inicial a $95^{\circ} \mathrm{C}$ por $10 \mathrm{mi}-$ nutos, 35 ciclos de $94^{\circ} \mathrm{C}-45$ segundos, $55^{\circ} \mathrm{C}$ 30 segundos, $72^{\circ} \mathrm{C}-30$ segundos, e 1 ciclo para extensão final de $72^{\circ} \mathrm{C}$ por 7 minutos. A Nested - PCR foi realizada utilizando uma mistura de reação contendo $1 \mathrm{X}$ Buffer, 2,5mM de $\mathrm{MgCl}_{2}$ , 0,4mM dNTP , 0,2pmol dos primers ED31 (5' - CCTCAGCCATTACACAGGCCTG TCCAAAG - 3'), e ED33 (5' - TTACAGTA GAAAAATTCCCCTC - 3') ${ }^{18}, 2,5 \mathrm{U}$ de Taq Recombinant DNA polimerase (Invitrogen) num volume total de reação de $50 \mu \mathrm{L}$. Adicionou-se, a seguir, $5 \mu \mathrm{L}$ do produto amplificado na primeira reação. A reação procedeuse com uma desnaturação inicial a $95^{\circ} \mathrm{C}$ por 10 minutos, 35 ciclos de $94^{\circ} \mathrm{C}-45$ segundos, $56^{\circ} \mathrm{C}$-30 segundos, $72^{\circ} \mathrm{C}-30$ segundos, e 1 ciclo para extensão final de $72^{\circ} \mathrm{C}$ por $7 \mathrm{minu}$ tos. O produto da PCR foi purificado utilizando-se o Kit Wizard SV Gel and PCR CleanUp System (Promega), obedecendo as especificações do fabricante.

A reação de marcação fluorescente foi realizada em microplaca de 96 poços, utilizando o Kit Big Dye (kit ABI Prisms ${ }^{T M}$ ), segundo especificações do fabricante. Foram realizadas duas reações para cada amostra, utilizando os primers ED31 e ED33. O produto da reação de marcação fluorescente foi precipitado e lavado utilizando-se uma solução de isopropanol a $65 \%$ seguida de etanol $60 \%$ gelado. O DNA foi desnaturado a $94^{\circ} \mathrm{C}$ por dois minutos e as amostras foram ressuspensas com uma solução de Loading Buffer e formamida e submetidas 
a uma eletroforese em gel de poliacrilamida 5\% desnaturante conduzida no aparelho ABI Prims ${ }^{T M} 377$ DNA Sequencer (Applied Biosystems) para seqüenciamento.

A seqüência consenso foi submetida a análise de subtipagem utilizando-se a ferramenta genotyping disponível on line pelo National Center for Biotechnology Information (NCBI) (http://www.ncbi. nih.gov/projects/genotyping/ formpage. cgi). A análise dos resultados obtidos foi realizada através de medidas descritivas (percentual) e teste estatístico de qui-quadrado $\left(\mathrm{X}^{2}\right)^{19}$. O estudo de associações entre os genótipos do HCV e subtipos do HIV1 foi realizado utilizando-se o teste de Goodman para contrastes entre e dentro de populações multinomiais ${ }^{20,21}$.

\section{Resultados}

A freqüência de co-infecção HIV/HCV no grupo de 150 pacientes analisados foi de $14,7 \%$.

A distribuição de freqüência dos genótipos e subtipos virais em pacientes mono e co-infectados pelo HIV / HCV está representada na Tabela 1.

Em virtude de limitações das técnicas empregadas não foi possível a determinação dos subtipos HIV em 18,6 \% em monoinfectados e $9,1 \%$ dos co-infectados, o mesmo ocorrendo com o HCV, onde não foi possível a determinação dos subtipos em $6,3 \%$ dos mono-infectados.

O estudo de associações entre os genótipos do HCV e subtipos do HIV-1 esta resumido na Tabela 2 .

\section{Discussão}

A co-infecção HIV/HCV nas amostras estudadas foi de 14,7\%. Estimativas mundiais segundo alguns autores variam, entre $4 \%$ e $90 \%$ dependendo da via de transmissão ${ }^{22}$.

Tabela 1 - Distribuição dos genótipos e subtipos virais em pacientes mono e co-infectados por HIV -1 / HCV em Botucatu e região

Table 1- Genotype and subtype virus distributions in patients mono and co-infected by HIV-1 / HCV in Botucatu and neighboring areas

\begin{tabular}{|c|c|c|c|}
\hline Tipos do HIV & Subtipos do HIV & Mono-Infectados & Co-Infectados \\
\hline \multirow[t]{4}{*}{$M$} & $B$ & $106(85,5 \%)$ & $17(85,0 \%)$ \\
\hline & $\mathrm{F}$ & $16(12,9 \%)$ & $3(15,0 \%)$ \\
\hline & $\mathrm{B} / \mathrm{F}$ & $2(1,6 \%)$ & 0 \\
\hline & Total & $124(100 \%)$ & 20 (100\%) \\
\hline Genótipos do HCV & Subtipos & Mono-Infectados & Co-Infectados \\
\hline \multirow[t]{5}{*}{1} & $1 \mathrm{a}$ & $124(25 \%)$ & $8(36,3 \%)$ \\
\hline & $1 b$ & $146(29,4 \%)$ & $6(27,3 \%)$ \\
\hline & $1 a / 1 b$ & $18(3,6 \%)$ & $2(9,1 \%)$ \\
\hline & não definido & $24(4,9 \%)$ & 0 \\
\hline & Total & $312(62,9 \%)$ & $16(72,7 \%)$ \\
\hline \multirow[t]{4}{*}{2} & $2 a / 2 c$ & 0 & 0 \\
\hline & $2 b$ & $3(0,6 \%)$ & 0 \\
\hline & não definido & $6(1,2 \%)$ & 0 \\
\hline & Total & $9(1,8 \%)$ & 0 \\
\hline \multirow[t]{4}{*}{3} & $3 a$ & $173(34,9 \%)$ & $6(27,3 \%)$ \\
\hline & $3 b$ & 0 & 0 \\
\hline & $3 c$ & 0 & 0 \\
\hline & Total & $173(34,9 \%)$ & $6(27,3 \%)$ \\
\hline \multirow[t]{4}{*}{5} & $5 a$ & $1(0,2 \%)$ & 0 \\
\hline & não definido & $1(0,2 \%)$ & 0 \\
\hline & Total & $2(0,4 \%)$ & 0 \\
\hline & Total & 496 (100\%) & 22 (100\%) \\
\hline
\end{tabular}


Tabela 2 - Associação entre os subtipos do HIV e genótipos do HCV em pacientes co-infectados $\mathrm{HIV} / \mathrm{HCV}$

Table 2 - Association between HIV subtype and HCV genotype in HIV/HCV co-infected patients

\begin{tabular}{lccc}
\hline & \multicolumn{2}{c}{ Subtipos } & HIV-1(Subtypes)( $\mathrm{n}=20)$ \\
Genótipos HCV (Genotypes)(n=20) & B $(\%)$ & $\mathrm{F}(\%)$ & Total \\
\hline $\mathbf{1}$ & $13(92,86)$ a B & $1(7,14)$ a A & 14 \\
$\mathbf{3}$ & $4(66,67)$ a A & $2(33,33)$ a A & 6 \\
\hline
\end{tabular}

Letras minúsculas representam comparações entre grupos (subtipos HIV-1 e genótipos HCV); letras maiúsculas representam comparações de respostas dentro de um mesmo grupo. A letra " $B$ " indica predominância em relação a " $A$ "

A subtipagem do HIV pôde ser concluída em 124 das 150 amostras analisadas, por limitação da técnica empregada. A ausência de amplificação pode ser explicada pela intensa taxa de mutação do HIV a cada ciclo replicativo, as quais não são corrigidas pela ausência de atividade de reparo. Muitas vezes, estas mutações têm lugar no sítio de anelamento dos primers, impossibilitando assim a amplificação por PCR. As seqüências de má qualidade ocorrem pela coexistência de populações virais, o que impossibilita seu seqüenciamento na ausência de outras abordagens técnicas capazes de isolar as diferentes cepas, como a clonagem.

A distribuição dos subtipos HIV em pacientes mono-infectados na região de Botucatu é semelhante aos relatados em outros trabalhos brasileiros, mostrando que o subtipo B ainda é o prevalente seguido pelo $\mathrm{F}^{23-25}$. Nos co-infectados o padrão de distribuição de subtipos do HIV-1 é semelhante aos mono-infectados, ou seja, predominância do subtipo B (85,0\%), seguido do F (15,0\%). A ausência de recombinantes $\mathrm{B} / \mathrm{F}$ pode estar relacionada à sua baixa freqüência na população e/ ou ao reduzido número de co-infectados encontrados neste estudo. A análise estatística aponta para uma tendência de aumento do subtipo F no grupo dos coinfectados.

A distribuição dos genótipos HCV em mono-infectados da região de Botucatu segue o mesmo padrão do Estado de São Pau$l^{26}$ e de outros estados brasileiros ${ }^{27-29}$, ou seja, uma maior freqüência do genótipo 1 seguido pelos 3 e 2, observando-se também a presença dos genótipos $5^{26,27,30}$. Comparando-se a freqüência dos genótipos $\mathrm{HCV}$ entre pacientes mono e co-infectados verificamos que nos co-infectados houve um aumento de $10 \%$ no genótipo 1 , uma queda de 7,7\% do genótipo 3 e ausência de outros genótipos. Outros autores encontraram em co-infectados HIV/HCV uma freqüência entre $40-55 \%$ do genótipo 1, 0,4-1,7\% do genótipo 2, 20-30\% do genótipo 3, 9-19\% do genótipo $4^{15,16,31}$. No entanto, a análise estatística pelo método de qui-quadrado $\left(\mathrm{X}^{2}\right)$ mostrou que o genótipo 2 sofreu maior influência das duas variáveis (mono-infecção e co-infecção)

$\mathrm{O}$ aumento na freqüência do genótipo 1 em co-infectados é motivo de preocupação, pois o tempo de tratamento preconizado para este genótipo é mais prolongado (12 meses) apresentando também uma pior resposta terapêutica ${ }^{5}$.

Quando se considera a freqüência dos subtipos do HCV em co-infectados, os achados atuais são contraditórios. Alguns autores ${ }^{15}$ encontraram uma freqüência para o subtipo $1 \mathrm{a}(36,3 \%)$ semelhante à obtida neste estudo enquanto outros autores vêm descrevendo freqüências inferiores para os subtipos $1 \mathrm{a}(24,1 \%), 1 \mathrm{~b}$ (11,2\%), 1a/1b $(6,9 \%)^{16}$.

O estudo de associação entre parâmetros constantes de Goodman não mostrou diferenças significativas entre os genótipos do HCV, pois numa comparação entre os grupos todas as categorias refletem uma situação de igualdade. Por outro lado, o comportamento dos subtipos do HIV-1 não foi semelhante, ou seja, no genótipo 1 (HCV) ocorreu predominância do subtipo B (HIV-1) e no 
genótipo 3 (HCV) a distribuição dos subtipo B e F do HIV-1 foi casual; no entanto, o tamanho da amostra limita de certa forma as con- clusões relativas às associações entre subtipos do HIV e genótipos do HCV, o que justifica novas investigações a esse respeito.

\section{Referências}

1. Babik JM and Holodniy M. Impact of highly active antiretroviral therapy and immunologic status on hepatitis $\mathrm{C}$ virus quasispecies diversity in human immunodeficiency virus/ hepatitis C virus-coinfected patients. J Virol 2003; 77(3): 1940-50

2. Di Bisceglie AM. Hepatitis C. Lancet 1998; 351: 351-5.

3. Domingo E, Escarmis C, Sevilla N, Moya A, Elena SF, Quer J, et al. Basic concepts in RNA viruses evolution. FASEB J 1996; 10: 859-64.

4. Nadler J. AIDS: etiologia. In: Veronesi R, Foccacia SR, ed. Tratado de Infectologia. São Paulo: Atheneu; 1996. p. 836.

5. Secretaria de Estado da Saúde. Manual de vigilância epidemiológica. Hepatites virais: normas e instruções. São Paulo; 2000.

6. Silva MD. Hepatite $\mathrm{C}$ e testes diagnósticos. Cad Farmácia 2001; 17(2): 111-5.

7. Maier I, Wu GY. Hepatitis C and HIV co-infection: a review. World J Gastroenterol 2002; 8(4): 577-9.

8. Rosado R R, Olmeda M P, Samaniego J G, Soriano V. Management of hepatitis $\mathrm{C}$ virus in HIV-infected persons. Ant Res 2001; 52: 189-98.

9. Pockros PJ. HCV in setting of HIV Co-infection. Med Writers' Circle. [cited 2003]. Disponível em: http:// www.hcvadvocate.org/hcsp/articles/Pockros-3.html. Acessado em 06 de setembro de 2005.

10. Vento S, Garofano T, Renzini C, et al. Enhancement of hepatitis $\mathrm{C}$ virus replication and liver damage in HIV coinfected patients on antiretroviral combination therapy. AIDS 1998; 12: 116-7.

11. Starcich BR, Hahn BH, Shaw GM, Mcneely PD, Modrow S, Wolf $\mathrm{H}$, et al. Identification and characterization of conserved and variable regions in the envelope gene of HTLV-III/LAC, the retrovirus of AIDS. Cell 1986; 45: 637 48.

12. Smith DB , Pathirana S, Davidson F, Lawlor E, Power J, Yap PL et al. The origin of Hepatitis C virus genotypes. $J$ Gen Virol 1977; 78: 321-8.

13. Okamoto H, Kurai K, Okada S-I,YamamotoK, Iizuka H, Tanaka T, et al. Full-length sequence of a hepatitis $\mathrm{C}$ virus genome having poor homology to reported isolates: comparative study of four distinct genotypes. Virology 1992; 188: 331-41.

14. Asten L van \& Prins M. Infection with concurrent multiple hepatic $\mathrm{C}$ virus genotype is associated with faster HIV disease progression. AIDS 2004; 18: 2319-24.
15. Ramos PC, Pascual CA, Arazo GP, Aguirre EJM, Lassierra $\mathrm{P}$. Clinical implications of hepatitis $\mathrm{C}$ virus genotype in human immunodeficiency virus infected patients with chronic liver disease. Rev Clin Esp 1998; 198(4) :212-6.

16. Piroth L, Bourgeois C, Dantin S, Waldner A, Grappin M, et al. Hepatitis $\mathrm{C}$ virus (HCV) genotype does not appear to be a significant prognostic factor in HIV-HCV-coinfected patients. Lippincott W\&WInc 1999; 13(4): 523.

17. Neau D, Jouvencel C, Legrand E, Trimoulet P, Galperin T, Chitty I, et al. Hepatitis C Virus Genetic Variability in 52 Human Immunodeficiency Virus-Coinfected Patients. $J$ M Virol 2003; 71: 41-8.

18. Mullins JI. HIV-1 HMA Subtyping Kit. [cited 2004 Mar]. Disponível em http://ubik.microbiol.washington.edu/ $\mathrm{HMA} / \mathrm{html} /$ primers.html. Acessado em 21 de janeiro de 2005.

19. Norman GR, Streiner Dl. Biostatistics - the pare essentials. Saint Louis ed Mosby ear book 1994. p. 260.

20. Goodman LA. Simultaneous confidence intervals for contrasts among multinomial populations. Ann Math Stat 1964; 35: 716-25.

21. Goodman LA. On simultaneous confidence intervals for multinomial proportions. Technometrics 1965; 7: 247-54.

22. Mendes-Corrêa MCJ \& Barone A. Hepatite C in patients co-infected with human immunodeficiency. A review and experience of a Brazilian ambulatory. Rev Inst Med Trop 2005; 47(2).

23. Galvão-Castro B, Couto-Fernandez JC, Mello MA Linhares-de-Carvalho MI, Castello-Branco LR, Bongertz $\mathrm{V}$, et al. A nationwide effort to systematically monitor HIV-1 diversity in Brazil: preliminary results. Brazilian Network for the HIV-1 Isolation and Characterization. Mem Inst Oswaldo Cruz 1996; 91: 335-8.

24. Tanuri A, Swanson P, Devare S, Berro OJ, Savedra A, Costa LJ. HIV-1 Subtypes Among Blood Donors from Rio de Janeiro, Brazil. J Acquir Immune Defic Syndr Hum Retrovirol 1999; 20: 60-6.

25. Vicente ACP, Otsuki K, Silva NB, Castilho MC, Barros FS, Pieniazek D, et al., The HIV epidemic in the Amazon basin is driven by prototypic and recombinant HIV-1 subtypes B and F. JAIDS 2000; 23: 327-31.

26. Spina AMM, Souza AT, Fernandes RB, Gushiken EKK, Ferraz M. Corvino SM, et al. Hepatitis $C$ virus genotypes in the state of Sao Paulo, Brazil. Simpósio de Hepatite C e vírus relacionados 2005: Montreal, Canadá. 
27. Bassit L, Ribeiro dos Santos G, Da Silva LC, Takei K Villaca P, David Neto E, et al. Genotype distributions of hepatitis $C$ virus in São Paulo, Brazil: rare subtype found. Hepatology 1999; 29: 994-5.

28. Oliveira MLA, Bastos FI, Sabino RR, Paetzold U, Schreier E, Pauli G,et al. Distribution of HCV genotypes among different exposure categories in Brazil. Braz J Med Biol Res 1999; 2: 79-282.

29. Paraná R, Vitvitski L, Berby F, Portugal M, Cotrim HP, Cavalcanti A. et al. HCV infection in northeastern Brazil: unexpected high prevalence of genotype $3 a$ and absence of African genotypes. Arq Gastroenterol 2000; 37: 213-6.
30. Lampe E, Espírito-Santo MP, Oliveira JM, Oliveira RV, Fialho MV, DeAlmeida AJ, et al. Genomic variability of hepatitis $C$ vírus strains from states of Bahia, Goiás and Rio de Janeiro, Brazil. J Braz Soc Virol 2004; 9(1): 156.

31. Rubio M, Rubio C, Nogués A, Manonelles A. Genotipos del virus de la hepatitis C. Estúdio de 302 pacientes coinfectados por el vírus de la inmunodeficiencia humana. Med Clin 2001; 116: 650-1.

Recebido em: 23/04/07 Versão final reapresentada em: 30/08/07

Aprovado em: 24/09/07 